\title{
Electron correlation, geometry, and energy spectrum of quadruply excited states
}

\author{
Yannis Komninos and Cleanthes A. Nicolaides \\ Theoretical and Physical Chemistry Institute, National Hellenic Research Foundation, \\ 48 Vas. Constantinou Avenue, 11635 Athens, Greece \\ (Received 31 January 1994; revised manuscript received 12 April 1994)
}

\begin{abstract}
There is a class of quadruply excited states of ${ }^{5} S^{o}$ symmetry where electronic motion is highly correlated and where the electrons tend to form a tetrahedron as the excitation energy increases toward the four-electron ionization threshold. This conclusion has been reached following ab initio state-specific calculations in Be for the lowest energy state of each intrashell manifold $n$, of the energies, the average radii $r_{n}$, and the average interelectronic angle $\theta_{12}$. In order to calculate $\theta_{12}$, a general theory is developed, applicable to arbitrary $N$-electron atomic states. The value of $\theta_{12}$ is straightforward to compute, and is given from a prescription transforming the expression for the two-electron interaction energy of the state to a formula for the probability density of $\cos \theta_{12}$. The state-specific calculations for each $n$, up to $n=6$, were done by the multiconfigurational Hartree-Fock method where all configurations with $n_{1}=n_{2}=n_{3}=n_{4}$ are included. For $n=3$, the main configuration $3 s 3 p^{3}$ has a weight of 0.90 while $\theta_{12}=103.3^{\circ}$. As $n$ increases, electron correlation increases relative to the Coulomb nuclear attraction. With increasing degeneracy, many configurations with high orbital angular momenta mix heavily, and $\theta_{12}$ increases. For example, for $n=6$, the $6 s 6 p^{3}$ configuration has a weight of only 0.59 and $\theta_{12}=106^{\circ}$. In this case, doubly, triply, as well as quadruply excited configurations with respect to $n s n p^{3}$ contribute to the wave function significantly. Finally, these four-electron ionization ladder states have a simple energy spectrum, given to a very good approximation by $E_{n}=-A^{\prime} / n^{2}\left(n^{1 / 2} \sim r_{n}\right)$, where $A^{\prime}$ is a constant. In conjunction with our earlier results on the geometry and the spectra of special classes of doubly and triply excited states, this finding leads to the conclusion that for highly correlated electronic motion the spectrum is dictated essentially by one dynamical variable, the average radius from the nucleus.
\end{abstract}

PACS number(s): $31.50 .+\mathrm{w}, 31.90 .+\mathrm{s}, 31.10 .+\mathrm{z}$

\section{INTRODUCTION}

The present paper presents theory and numerical results for the conceptual and quantitative understanding of quadruply excited states (QES) which can be grouped into a class characterized by a linear combination of configurations with $n=n_{1}=n_{2}=n_{3}=n_{4}$, where $n_{i}$ are the principal quantum numbers of the excited electrons. Specifically, by applying the state-specific approach to the understanding and computation of wave functions and properties of multiply excited states (MES), we show that there is a class of QES with parallel spins, ${ }^{5} S^{o}$ symmetry, whose electronic geometry, as computed from first principles, tends to that of a tetrahedron as the $E=0$ threshold is approached, and whose spectrum obeys a simple law, analogous to the ones that we derived previously for special classes of doubly excited states (DES) and for triply excited states (TES).

The determination of the geometric localization of the electronic distribution of these four-electron states, required the development of theory for the computation of the average value of the angle among the electrons. This theory is presented in Sec. IV.

\section{BACKGROUND: COMPUTATION, GEOMETRY AND ENERGY SPECTRUM OF CLASSES OF DOUBLY AND TRIPLY EXCITED STATES}

The spectra of atoms are determined by the excitations of electrons into states where the occupied shells and subshells have quantum numbers $n$ and/or $l$ that are larger than those of the ground state. There is an infinite number of possible excitations to states with different quantum numbers, and a variety of spectroscopies together with quantum theory and computation aim at creating reliable knowledge as regards the fundamental wave-function characteristics and the properties of individual states as well as of groups of states which can be assigned to classes with particular common features.

The simplest and the most extensively studied case of a class of states whose common features have been understood through a combination of experiment and theory, is the one-electron Rydberg series. This understanding is reflected in (1) the calculable function of the active electron for any symmetry and energy by solving appropriate quantum or semiclassical one-dimensional differential equations. (2) The hydrogenlike formula for the energy spectrum of each symmetry

$$
\left[E_{n}(\mathrm{Ry})\right]=-\frac{C}{\left(n^{*}\right)^{2}},
$$

where $n^{*}$ is an effective quantum number, with slight or strong energy dependence coming from the quantum defect which measures the deviation from the hydrogenic situation due to the presence of the core electrons, or of other Rydberg series, or of DES.

In the case of DES, the conceptual and computational simplicity of the one-electron picture is lost. Now, as regards the prediction of the observable properties, one 
must compute wave functions which are very extended and which contain quantitatively the self-consistent dynamical interelectronic interactions, the near degeneracies (which increase with excitation), and the remaining electron correlation. Furthermore, the DES appear in every $N$-electron system and not just in He-like ones, which thus far have constituted the object of the majority of investigations. Hence, in the $N$-electron case $(N>2)$, the complexity of electronic structure (e.g., many open subshells), and the multiplicity of interelectronic correlation effects (e.g., triple, products of pairs, core-valence, etc., virtual excitations), require the existence of general theory and practical methods for its implementation.

An approach to the problem of calculating the correlated wave functions and properties of MES, in position or in momentum space, with particular emphasis to the $a b$ initio treatment of special classes of DES and of TES where the zeroth order orbitals are assigned the same principal quantum number $n$ (intrashell configurations), has been presented by us in a series of articles (e.g., [1-6] and References therein). In spite of earlier discouraging statements as to the validity and calculability of HartreeFock (HF) or multiconfigurational (MCHF) wave functions for bound states in the continuum or as to the possibility of treating MES with strong correlations in terms of $\mathrm{O}(3)$ configurational expansions, the main direction in our research has been toward the computation of state-specific wave functions using methods developed for low-lying polyelectronic states. Thus the localized wave function $\Psi_{0}^{m}$ of each MES, $|m\rangle$, is written as

$$
\Psi_{0}^{m}=\Phi_{\mathrm{MCHF}}^{m}+X_{\mathrm{loc}}^{m},
$$

where $\Phi_{\mathrm{MCHF}}^{m}$ is the MCHF wave function and $X_{\text {loc }}^{m}$ stands for the remaining correlation configurations which are added by the variational optimization of analytic virtual orbitals. Our choice of the MCHF configurations is based on the anticipated character of each state of interest and on a strategy toward computational economy. As regards the method for obtaining the MCHF orbitals, our experience suggests that the numerical method [7] works well for the low-lying states but that analytic methods based on the iterative use of variational and of natural orbitals are more convenient for the high-lying ones $[1,5]$.

The theory is not restricted by the type of localized or quasilocalized state of interest or by the number of electrons. Furthermore, it has been employed for the $a b$ initio computation of DES not only of low energy but also of high energy, in neutral atoms and in negative and positive ions, thereby allowing us to draw conclusions on previously unexamined questions about the properties of two-electron states where electron correlation dominates $[1,2,5,8-10]$.

Returning to the problem of distinguishing special classes of DES and TES and computing their properties, the work in [1-5] produced the following results and conclusions which have been used as input to the present study:

(1) Correlated wave functions of certain symmetries where the zero-order MCHF description is given as a combination of all the intrashell configurations, $n=n_{1}=$ $n_{2}$ for DES and $n=n_{1}=n_{2}=n_{3}$ for TES, show local- ization tending to the rigid rotor for the DES and to the equilateral triangle for the TES as $n$ becomes large. The first case represents states on the "Wannier ridge" [11] and constitutes a two-electron ionization ladder (TEIL), while the second case represents TES on a "hyperridge," defining the class of the three-electron ionization ladder (THEIL) states. Both the angles and the average radii are calculated from first principles in terms of specially defined functions. In fact, these findings have proven useful for the adoption of a fixed-radius model for the qualitative analysis of the TES [12].

(2) Of all the possible states corresponding to configurations with $n_{1}=n_{2}$ or $n_{1}=n_{2}=n_{3}$, the states of the TEIL or the THEIL correspond to the lowest root of the Hamiltonian matrix constructed in these manifolds. This is the original result of [1] for the TEIL states. It is the lowest root, together with the conditional probability plots and the $a b$ initio evaluation of average radii and angles, that ought to be used for the identification of such localized states and not the simplistic reference to an intrashell single configuration (say the $n s^{2}$ for the ${ }^{1} S$ TEIL). In fact, we have found that, as $n$ increases the dominant configurations of the TEIL or THEIL states tend toward higher orbital momenta (e.g., see Table I of Ref. [4] for the $\mathrm{He}^{-2} S$ TEIL resonances).

(3) The results of geometrical localization and of lowest energy per intrashell manifold were used for the derivation of formulas for the excitation spectra of the TEIL and the THEIL states [2], which have the remarkably simple form, resembling Eq. (1):

$$
\begin{aligned}
E_{n}(\text { TEIL or THEIL }) & =-A_{n} \frac{n(n-1)}{r_{n}^{2}}, \\
& =-\frac{A^{\prime}}{n^{2}}(\text { for large } n),
\end{aligned}
$$

$A_{n}$ varies very slowly and $r_{n}$ is computed as an average value for each $n$. Equation (4) follows from Eq. (3) because our computations showed that the proportionality constant converges and that $r_{n}$ is proportional to $n^{2}$. It should be noted that by writing an energy-independent, integer quantum number in Eq. (4), we assume that the energy region of interest is not perturbed strongly by, say MES with $(N+1)$ number of electrons. (Incidentally, it might be interesting to study a TEIL spectrum which is perturbed by TES in $N$-electron ions.)

For the TEIL states, Eq. (3) reduces to the "double Rydberg" formula proposed by Read [13] empirically, from data on low-lying states, and subsequently analyzed and used by a number of authors who treat such states in terms of collective coordinates [14-20]. In the recent paper by Zhang and Rau [14], the comparison which is made among various results $[14,19,20]$ (Tables IV-VI), shows significant discrepancies.

\section{THEORY AND COMPUTATION OF THE FOUR-ELECTRON-IONIZATION-LADDER (FEIL) STATES OF ${ }^{5} S^{\circ}$ SYMMETRY IN Be}

We consider QES for $Z=4$, where all spins are parallel and the overall symmetry is ${ }^{5} S^{o}$. The configuration 
TABLE I. Mixing coefficients of the most important (coefficients equal to or larger than 0.05) intrashell configurations of the four-electron ionization ladder (FEIL) states in $\mathrm{Be}^{5} S^{o}$, obtained from MCHF calculations.

\begin{tabular}{|c|c|c|c|c|}
\hline Configuration & $n=3$ & 4 & 5 & 6 \\
\hline$n s n p^{3}$ & 0.95 & 0.87 & 0.81 & 0.77 \\
\hline$n s n p n d^{2}$ & 0.29 & 0.42 & 0.46 & 0.48 \\
\hline$n s n f n d^{2}$ & & 0.14 & 0.20 & 0.23 \\
\hline$n d n f n p^{2}$ & & -0.18 & -0.24 & -0.28 \\
\hline$n p n d n f^{2}$ & & 0.05 & 0.09 & 0.13 \\
\hline$n f n d^{3}$ & & -0.05 & -0.08 & -0.11 \\
\hline
\end{tabular}

corresponding to the lowest state of this symmetry is the $2 s 2 p^{3}$. The problem then is how to compute the wave functions and their energies as well as the electronic geometry of the intrashell QES corresponding to the lowest energy state of each manifold $n$. The method and its justification allowing the computation of correlated wave functions of MES are discussed in the previous section and in Refs. [1-6]. The theory which allowed the determination of the electronic geometry of these QES, and which is applicable to any $N$-electron atomic state, is given in the next section. Here we present our numerical results.

As regards the constitution of the MCHF correlated wave functions for each $n$, the most important configurations and their coefficients (equal to or larger than 0.05) are given in Table I. The calculations were done using linear combinations of all $n_{1}=n_{2}=n_{3}=n_{4}$ configurations for each manifold $n$. As in the case of doubly and triply excited states of the TEIL and THEIL spectra, as $n$ increases, that is, as energy and degeneracy increase and the relative importance of interelectronic interactions increases, the dominant single configuration $n s n p^{3}$ loses weight and even quadruply excited configurations acquire significance. The calculations stopped at $n=6$ because, as we have stated in the past, for larger $n$ there appear convergence difficulties with the Froese-Fischer numerical MCHF computer code [7] and because by the time we reach $n=6$, the results are sufficient to allow the deduction of conclusions.

The expectation values of these correlated wave functions are presented in Table II. Column 1 contains the total energies. The lowest state is about $266.4 \mathrm{eV}$ above the Be $1 s^{2} 2 s^{21} S$ ground state. Our experience with statespecific calculations of TEIL and THEIL states $[1,2,4,5]$ has shown that the effect of $X_{\text {loc }}$ [Eq. (2)] is small, and as $n$ increases it becomes essentially negligible. Therefore, in this work only the $\Phi_{\mathrm{MCHF}}^{n}$ and $E_{\mathrm{MCHF}}^{n}$ were com-
TABLE II. Results of calculations using the $\mathrm{Be}{ }^{5} S^{\circ}$ FEIL correlated wave functions. $r_{n}$ and $\theta_{n}$ represent the average distance and interelectronic angle respectively. $A_{n}$ and $A_{n}^{\prime}$ are defined by Eqs. (3) and (4). These results, in conjunction with the fact that Eq. (3) is decuced from the geometry of the oblate symmetric top (see text), show that in the limit of $E_{n} \rightarrow 0$, the four electrons leave the atom in the tetrahedral geometry. Energies and distances are given in atomic units.

\begin{tabular}{llrrrrr}
\hline \hline$n$ & $-E_{n}$ & \multicolumn{1}{c}{$r_{n}$} & $\frac{r_{n}}{n^{2}}$ & \multicolumn{1}{c}{$\theta_{n}$} & \multicolumn{1}{c}{$A_{n}$} & $\boldsymbol{A}_{n}^{\prime}$ \\
\hline 2 & 4.8743 & 1.72 & 0.43 & $99.5^{\circ}$ & 7.21 & 19.50 \\
3 & 2.2871 & 3.90 & 0.43 & $103.3^{\circ}$ & 5.80 & 20.60 \\
4 & 1.3173 & 6.95 & 0.43 & $105.2^{\circ}$ & 5.30 & 21.08 \\
5 & 0.8528 & 10.86 & 0.43 & $105.7^{\circ}$ & 5.03 & 21.32 \\
6 & 0.5962 & 15.55 & 0.43 & $106.0^{\circ}$ & 4.80 & 21.46 \\
\hline \hline
\end{tabular}

puted. Column 2 contains the average $r_{n}$. These values are smaller than those of the Li THEIL states [Table I of Ref. [2]] whose main configuration is $2 p^{34} S^{o}$. Column 3 demonstrates that $r_{n} \sim n^{2}$, a key feature of the structure of not only the one-electron atom but also of the TEIL and THEIL states $[1,2,4,5]$. Thus, together with the results of column 5 and of the corresponding energy spectrum (see below), it is seen that these states fall into a class with special properties. We call this class the four-electron ionization ladder (FEIL).

Column 5 contains the average value of the angle among pairs of electrons. The computation of this property for a many-electron system has not been done before and required the development of the necessary theory. This is given in the next section, together with an analysis of the related importance of electron correlation. The results of column 5 show that as $n$ increases, the angle tends to that of tetrahedral geometry $\left(109.5^{\circ}\right)$.

Finally, we have applied Eqs. (3) and (4) to the calculated results. The justification for doing so for the FEIL states in conjunction with the results of the oblate symmetric top is given in Ref. [2]. Indeed, these equations fit our computed energies very well and the corresponding proportionality constants $A_{n}$ and $A_{n}^{\prime}$ are given in columns 5 and 6 of Table II. As $n$ increases, $A_{n}^{\prime}$ converges to a constant value.

\section{THEORY AND COMPUTATION OF THE AVERAGE INTERELECTRONIC ANGLE $\theta_{12}$}

The general second-order density matrix, in its spinless form, is defined through the many-body wave function as

$$
\Gamma\left(\mathbf{r}_{1}^{\prime}, \mathbf{r}_{2}^{\prime} ; \mathbf{r}_{1}, \mathbf{r}_{2}\right)=\sum_{\sigma_{1}, \sigma_{2}, \ldots, \sigma_{n}} \int \Psi\left(\mathbf{r}_{1}^{\prime} \sigma_{1}, \mathbf{r}_{2}^{\prime}, \sigma_{2}, \mathbf{r}_{3} \sigma_{3}, \ldots, \mathbf{r}_{n} \sigma_{n}\right) \Psi^{*}\left(\mathbf{r}_{1} \boldsymbol{\sigma}_{1}, \mathbf{r}_{2} \sigma_{2}, \mathbf{r}_{3} \sigma_{3}, \ldots, \mathbf{r}_{n} \sigma_{n}\right) d \mathbf{r}_{3} \cdots d \mathbf{r}_{n}
$$

where summation over all spin variables is performed [21]. Its diagonal element is the probability density of simultaneously finding one particle at $\mathbf{r}_{1}$ and another at $\mathbf{r}_{2}$. In terms of the latter quantity, the energy of the two-particle interaction $f\left(r_{1}, r_{2}\right)$ can be written as [21]

$$
E=N(N-1) / 2 \int f\left(\mathbf{r}_{1}, \mathbf{r}_{2}\right) \Gamma\left(\mathbf{r}_{1}, \mathbf{r}_{2} ; \mathbf{r}_{1}, \mathbf{r}_{2}\right) d \mathbf{r}_{1} d \mathbf{r}_{2}
$$

where the factor $N(N-1) / 2$ is the number of electron pairs. 
Similarly, we define the probability that the position vectors of two particles form an angle $\theta_{12}$ as

$$
\rho\left(\cos \theta_{12}\right)=\int_{\theta_{12}=\text { const }} \Gamma\left(\mathbf{r}_{1}, \mathbf{r}_{2} ; \mathbf{r}_{1}, \mathbf{r}_{2}\right) d \mathbf{r}_{1} d \mathbf{r}_{2} .
$$

Let $\Psi$ be a linear combination of $N$-electron determinants over a set of orthonormal spin-orbitals. If two determinants differ by more than two spin-orbitals, their product does not contribute to $\varrho\left(\cos \theta_{12}\right)$. Otherwise, their con- tribution is computed according to the Slater-Condon rules [22]. In general, the calculation of $\varrho\left(\cos \theta_{12}\right)$ requires the evaluation of the following expression over four orbitals:

$$
\int_{\theta_{12}=\text { const }} u_{a}\left(\mathbf{r}_{1}\right) u_{b}\left(\mathbf{r}_{2}\right) u_{c}\left(\mathbf{r}_{1}\right) u_{d}\left(\mathbf{r}_{2}\right) d \mathbf{r}_{1} d \mathbf{r}_{2} .
$$

Separating radial and angular parts, this is written as

$$
\left\langle R_{a} \mid R_{c}\right\rangle\left\langle R_{b} \mid R_{d}\right\rangle \int_{\theta_{12}=\mathrm{const}} Y_{l_{a} m_{a}}\left(\theta_{1}, \varphi_{1}\right) Y_{l_{b} m_{b}}\left(\theta_{2}, \varphi_{2}\right) Y_{l_{c} m_{c}}^{*}\left(\theta_{1}, \varphi_{1}\right) Y_{l_{d} m_{d}}^{*}\left(\theta_{2}, \varphi_{2}\right) d \hat{\mathbf{r}}_{1} d \hat{\mathbf{r}}_{2} .
$$

As a function of $\theta_{12}$, the integral can be expanded in terms of the orthogonal Legendre polynomials $P_{k}\left(\cos \theta_{12}\right)$. After some straightforward algebra, the coefficients of this expansion, expressed in terms of $3-j$ symbols, are found to be

$$
\begin{aligned}
c_{k}= & \delta_{m_{a}+m_{b}, m_{c}+m_{d}} \sqrt{\left(2 l_{a}+1\right)\left(2 l_{b}+1\right)\left(2 l_{c}+1\right)\left(2 l_{d}+1\right)}(-1)^{m_{b}+m_{c}} \\
& \times \frac{2 k+1}{2}\left(\begin{array}{ccc}
l_{a} & l_{c} & k \\
0 & 0 & 0
\end{array}\right)\left(\begin{array}{ccc}
l_{b} & l_{d} & k \\
0 & 0 & 0
\end{array}\right)\left(\begin{array}{ccc}
l_{a} & l_{c} & k \\
m_{a} & -m_{c} & -m_{a}+m_{c}
\end{array}\right)\left(\begin{array}{ccc}
l_{b} & l_{d} & k \\
m_{b} & -m_{d} & -m_{b}+m_{d}
\end{array}\right) .
\end{aligned}
$$

Apart from the factor $(2 k+1) / 2$, these coefficients are the same as the ones appearing in the expression of the Coulomb interaction matrix elements in terms of the $R^{k}$ integrals. In this case, the integral over three spherical harmonics appears due to the expansion of the two-body operator $r_{12}^{-1}$ in terms of the Legendre functions. Comparison of the two cases gives this correspondence

$$
R^{k}(a, b ; c, d) \Rightarrow\left\langle R_{a} \mid R_{c}\right\rangle\left\langle R_{b} \mid R_{d}\right\rangle \frac{2 k+1}{2} P_{k}\left(\cos \theta_{12}\right) .
$$

Thus, an expression of the Coulomb interaction can be transformed into an expression of $\varrho\left(\cos \theta_{12}\right)$ by the substitution (11). Therefore, $\varrho\left(\cos \theta_{12}\right)$ of each state is computable and is directly related to the two-electron interaction energy expression of this state, either in the independent particle approximation or with electron correlation included. We note that when prescription (11) is followed, division of the energy expression by the number of electron pairs is necessary in order that the normalization condition

$$
\int_{0}^{\pi} \rho\left(\cos \theta_{12}\right) \sin \left(\theta_{12}\right) d \theta_{12}=1
$$

is satisfied, as it is shown by comparing (6) and (7).

In practice, because of the orthonormality condition of the Legendre polynomials and since $P_{1}(\cos \theta)=\cos \theta$, the expected value $\left\langle\cos \theta_{12}\right\rangle$ for a single configuration is proportional to the coefficient of the $G^{1}$ integral (divided by the number of electron pairs). Thus if a $G^{1}$ integral is absent, the expected value of $\cos \theta_{12}$ is 0 (i.e., $\theta_{12}$ is $\pi / 2$ ). In the present case of the $\mathrm{Be}^{5} S^{o}$ state, the expectation value of $\theta_{12}$ from just the $2 s 2 p^{3}$ configuration is $99.5^{\circ}$. The main contributor to the opening of the angle for the first few TEIL states is the nsnpnd $d^{2}$ configuration (Table I) because of its large mixing coefficient and because of its large $R^{1}$ integral with $n s n p^{3}$. Note that because of the overlaps in (11), correlation corrections with virtual orbitals where $n^{\prime} \neq n$ contribute much less to the $\varrho\left(\cos \theta_{12}\right)$ of an atomic state than the intrashell angular correlations.

\section{CONCLUSIONS}

The state-specific approach to the computation and analysis of multiply excited states [1-6], in conjunction with the theory of Sec. IV for the calculation of interelectronic average angles for arbitrary $N$-electron atomic states, was applied to intrashell quadruply excited states of Be of ${ }^{5} S^{o}$ symmetry for $n=2$ to 6 . Following the theory and results of [1-5] on special classes of doubly and triply excited states, we focused on the properties of the state of lowest energy of each manifold $n$. These properties turned out to be $n$ dependent in a regular manner. As $n$ increases, i.e., as we enter the regime where the interelectronic correlations dominate and the Coulomb attraction is diminished, two remarkable results emerge clearly.

The first is that, as a result of the mixing of many intrashell configurations with self-consistently obtained orbitals, the average angle between the electrons opens up, tending to that of a tetrahedron (Table II). This finding can be added to the ones for the TEIL $[1,2]$ and THEIL [2] states, where the linear and equilateral triangle geometries are approached as $n$ increases.

The second is that Eq. (4) is also satisfied for the FEIL states. In other words, two results from the physics of the hydrogen atom, the $1 / n^{2}$ dependence of the energy spectrum and the $r_{n} \sim n^{2}$ proportionality, are shown to be valid also in special classes of doubly, triply, and quadruply excited states. The common feature of these states is that they represent situations where, for each value of $n$ the interelectronic correlations are maximal, regardless of the number of electrons. 
[1] Y. Komninos and C. A. Nicolaides, J. Phys. B 19, 1701 (1986).

[2] C. A. Nicolaides, M. Chrysos, and Y. Komninos, Phys. Rev. A 39, 1523 (1989); J. Phys. B 21, L73 (1988); Phys. Rev. A 41, 5244 (1990).

[3] C. A. Nicolaides and Y. Komninos, J. Phys. B 23, L571 (1990).

[4] M. Chrysos, G. Aspromallis, Y. Komninos, and C. A Nicolaides, Phys. Rev. A 46, 5789 (1992).

[5] Y. Komninos, S. Themelis, M. Chrysos, and C. A. Nicolaides, Int. J. Quantum Chem. S 27, 399 (1993).

[6] C. A. Nicolaides, N. Makri, and Y. Komninos, J. Phys. B 20, 4963 (1987); C. A. Nicolaides, N. Piangos, and Y. Komninos, Phys. Rev. A 48, 3578 (1993); C. A. Nicolaides and S. I. Themelis, J. Phys. B 26, L387 (1993); C. A. Nicolaides, ibid. 26, L291 (1993).

[7] C. Froese-Fischer, The Hartree-Fock Method for Atoms (Wiley, New York, 1977); Comput. Phys. Commun. 14, 145 (1978).

[8] D. R. Herrick and O. Sinanoglu, Phys. Rev. A 11, 97 (1975); O. Sinanoglu and D. R. Herrick, J. Chem. Phys. 62, 886 (1975).

[9] D. R. Herrick, Adv. Chem. Phys. 52, 1 (1982).
[10] G. S. Ezra and R. S. Berry, Phys. Rev. Lett. 52, 1252 (1984); C. D. Lin, ibid. 52, 1253 (1984).

[11] U. Fano, Rep. Prog. Phys. 46, 97 (1983).

[12] C.-G. Bao, J. Phys. B 25, 3725 (1992).

[13] F. H. Read, J. Phys. B 12, 449 (1977); 23, 951 (1990).

[14] A. R. P. Rau, J. Phys. B 16, L699 (1983); L. Zhang and A. R. P. Rau, Phys. Rev. A 46, 6933 (1992).

[15] C. D. Lin and S. Watanabe, Phys. Rev. A 35, 4499 (1987).

[16] H. Wang, J. Phys B 19, 3401 (1986).

[17] I. K. Dmitrieva and G. I. Plindov, J. Phys. B 21, 3055 (1988).

[18] Q. Molina, Phys. Rev. A 39, 3298 (1989).

[19] N. Koyama, A. Takafuji, and M. Matsuzawa, J. Phys. B 22, 553 (1989).

[20] J. M. Rost and J. S. Briggs, J. Phys. B 21, L233 (1988); 22, 3587 (1989).

[21] N. H. March, W. H. Young, and S. Sampanthar, The Many Body Problem in Quantum Mechanics (Cambridge University Press, Cambridge, England, 1967).

[22] J. C. Slater, Quantum Theory of Atomic Structure (McGraw-Hill, New York, 1960). 